\title{
Evaluation of Cesarean Section Scar Using Maternal Serum Level of Creatine Kinase Isoenzyme (Ck-Mb) Prior to Delivery
}

\section{Original Article}

\author{
Asmaa I. Mohamed, Rania M. Abdel Wahed, Iman B. AbdRabou \\ Department of Obstetrics and Gynecology, Faculty of Medicine For Girls, Al-Azhar University, \\ Cairo, Egypt
}

\begin{abstract}
Aim: This study aimed to evaluate cesarean scar by using maternal serum creatine kinase isoenzyme (CK-MB) prior to delivery as a soft marker to detect CS complications (uterine rapture, dehiscence).

Material and Methods: It was a prospective study carried out at Alzahraa University Hospital where 90 pregnant women divided into two groups : group A included 60 women with previous cesarean section. They were subdivided into 2 subgroups : group (A1) 19 patients who included cases with emergency cesarean delivery and group (A2) 41 patients which included cases with elective cesarean delivery. Group B includced the control group which included 30 patients with planned normal vaginal deliveries. Before delivery blood sample were collected from both groups before cesarean delivery and during normal vaginal delivery. The plasma (heparinized/EDTA) was collected from our 90 subject. Total CK, CK-MB level analyses were done by immuno-inhibition method using auto- analyzer. The normal values of CK total (24-170 U/L) and CKMB (0-25U/L).

Results: Twenty patients had scar abnormality (defect=4,thin=16) and remaining forty cases had intact scar these patients had elevated levels of CKMB with sensitivity, specificity, PPV and NPV for thin scar 100\%, 84.09\%, 69.6\% and 100\%, respectively, and sensitivity, specificity, PPV and NPV for rapture scar 100\%,78\%,38.9\% and 100\%, respectively.

Conclusion: CKMB can be used as a soft marker beside other methods for detection of cesarean scar abnormality (uterine rapture, dehiscence ) prior to delivery with high sensitivity and specificity.
\end{abstract}

Received: 17 April 2020, Accepted: 26 October 2020

Key Words: Cesarean delivery, cesarean section, CKMB, uterine dehiscence, uterine rapture

Corresponding Author: Asmaa I. Mohamed, Department of Obstetrics and Gynecology Faculty of Medicine For Girls Al-Azhar University, Cairo, Egypt, Tel.: 01033446324, E-mail: eltobgyasmaa4@gmail.com

ISSN: 2090-7625, August 2021, Vol.11, No. 3

\section{INTRODUCTION}

Cesarean section is the commonest obstetric operation performed and one of the commonest surgical procedures in general. It is performed as both elective and urgent. However, as a surgical procedure, there are risks of complications and overuse can be harmful to both mothers and neonates ${ }^{[1]}$, one of these complications, Cesarean scar defect. Because of the stretching of the muscle during pregnancy or the strong contractions of labor, the old cesarean scar might not stand the strain of labor and it becomes thin or begins to separate. When it does, it is called scar dehiscence. Rarely, the scar opens and extends into other parts of uterus. This is called uterine rupture ${ }^{[2]}$ Uterine rupture can lead to many complications such as postpartum hemorrhage, hysterectomy, even maternal death and intrapartum/infant deaths, and post-hypoxic encephalopathy and admissions to NICU ${ }^{[3]}$.

However, The inability to precisely confirm the integrity of scarred lower uterine segment (LUS) becomes the indication of a repeat cesarean section ${ }^{[2]}$. But there is range of error during the sonographic measurement because many factors can affect the Lower uterine segment measurement (eg: contractile state of uterus, displacement of amniotic fluid, fetal movement and position, operator's pressure through the transducer during the examination and fullness of bladder ${ }^{[4]}$.

Creatine kinase (CK) is a key enzyme of energy metabolism, especially in muscle tissue. CK has two polypeptide chains of $\mathrm{M}$ and $\mathrm{B}$, and three isomers, $\mathrm{CK}$ BB, CK-MB and CK- MM. Creatine Kinase can also be elevated in absence of neuromuscular diseases or cardiac injury, CK and its isoenzyme activity show a great variation during pregnancy. Similar trends were observed for the CK isoenzymes $\mathrm{CK}-\mathrm{MB}^{[5]}$.

Several reports indicate elevated activity of serum $\mathrm{CK}$ in the maternal blood during child birth, The uterus and placenta, two organs which were reported to embody substantial amounts of these enzymes, and which participate actively in the process of labor, are thought to release these enzymes to the circulation during labor ${ }^{[6]}$. 


\section{PATIENTS AND METHODS}

A prospective study performed at Al-Zahraa University Hospital, where 90 pregnant women between 18 and 40 years old attending Al-Zharaa Outpatient Clinic and emergency ward, were included. These 90 subjects were divided into two groups; group A which included 60 women with previous cesarean section. They were subdivided into 2 subgroups; group A1 included 19 patients which included cases with emergency cesarean delivery and they were enrolled from emergency ward and group A2 included 41 patients which included cases with elective cesarean delivery and they were enrolled from outpatient clinic. Group B; the control group which included 30 patients with planned normal vaginal deliveries. Both primigravida and multigravida control cases, they were enrolled from both emergency ward and from outpatient clinic.

Image acquisition: Before delivery, routine fetal US was done for every case included fetal measures and biophysical profiles. Sonographic examination was done by Voluson 730 Pro V machine equipped with a 3.5-5- $\mathrm{MHz}$ transabdominal and 5.5-9- $\mathrm{MHz}$ transvaginal probe. Ultrasound examinations were performed by designated obstetrician sonologists who were not involved in management of the cases, the lower uterine segment was assessed by transabdominal route by 2D US. Transabdominal evaluation was performed with the women in a supine position with a comfortably full bladder (we avoided over distended bladder to avoid stretching of the lower uterine segment), to measure the whole thickness of the anterior wall of the lower uterine segment where it covers the fetal head, as follow, (one cursor positioned at urine-bladder interface and the other at decidua-amniotic fluid interface).

CKMB:Then blood sample were collected from both groups before cesarean delivery and during normal vaginal delivery. The serum or plasma (heparinized/EDTA) was collected from our 90 subject. The serum was separated from blood and were put in epindoorf tubes and sent immediately for analysis. Total CK, CK-MB level analyses were done by Immuno-inhibition method using autoanalyzer. The normal values of CK total (24-170 U/L) and CKMB (0-25U/L).

Intraoperative : In women who had elective and urgent cesarean section the actual measurement of LUS thickness was recorded using a Vernier caliper according to, technique. Thickness of the lower flap of LUS at center was measured after delivery of fetal head as follows: after uterine incision, open the movable jaw of a sterile vernier caliper away from the fixed jaw through the screw. The lower flap of the LUS is fixed in between the tips of the outer jaws of the caliper, without tissue compression, then, remove the jaw and take the reading. Measurements were used for correlation with sonograically measured LUS thickness and CKMB values.

\section{STATISTICAL ANALYSIS}

Analysis of data was performed using SPSS v. 25 (Statistical Package for Scientific Studies) for Windows and Med Calc v. 18. Quantitative data were expressed as mean \pm standard deviation (SD). Qualitative data were expressed as frequency and percentage. Sensitivity, specificity, positive predictive value (PPV) and negative predictive value (NPV) were calculated. Pearson correlation analysis was performed to determine changes in CKMB and its relation to scar integrity. Results were expressed in the form of correlation coefficient (R) and P-values. $P$ value less than 0.05 was considered statistically significant.

\section{RESULTS}

Ninety pregnant women were included, sixty with previous cesarean delivery and forty with vaginal delivery. Our age groups were ranged from 18-40 years old with mean age for 30.53 for CS and 27.87 for NVD with SD 5.20, 5.83, respectively.

In our study, G0 numbers were three $(10 \%), \mathrm{G} 2$ numbers in CS were $21(35 \%)$ and in NVD were $10(33.3 \%), \mathrm{G} 3$ numbers in CS were $22(36.7 \%)$ and in NVD were $6(20 \%)$, G4 numbers in CS were $10(16.7 \%)$ and in NVD were $5(16.7 \%)$, G5 numbers in CS were $3(5 \%)$ and in NVD were $4(13.3 \%)$, G6 numbers in CS were $3(5 \%)$ and in NVD were $1(3.3 \%), \mathrm{G} 7$ numbers in CS were $1(1.7 \%)$ and in NVD were $1(3.3 \%)$ (Table 1).

The scar intraoperative was classified into four categories; number of defector raptured scar 4 (6.7\%), intact scar $39(65 \%)$, thick scar $1(1.7 \%)$ and thin scar16 (26.7\%) (Table 2).

CKMB was higher in CS group with mean 27.17 and SD 17.34 and was normal i.e. not affected in NVD group with mean 19.42 and SD 6.79 (Figure 1).

Intraoperative CKMB level was found to be high level in scar defect with mean value 39.60, SD value 9.02 and in thin scar with mean value 44.39 and SD 13.82, it was found to be normal in cases with intact and thick scar with mean value 19.04 and 18.70 , respectively. There is statistically significant difference of mean CKMB in CS group according to scar integrity distribution by ANOVA test $(p .<0.05)$. By doing Student-Newman-Keuls test; Posthoc test that is used for pairwise comparison of subgroups, the significant difference appears between cases with defect and intact scar and between cases with intact and thin scar.as shown in (Table 3, Figure 2) 
Intraoperative CKMB was found to be high in both raptured and thin scar by using vernier caliper with mean value 41.013 and 43.63 ,respectively. It was found to be normal in thick scar by using the same caliper measuring 43.63. (Table 4, Figure 3).

There is statistically significant difference of mean CK MB in CS group according to Vernier caliper distribution by ANOVA test $(p .<0.05)$. By doing Student-Newman-Keuls test; Post-hoc test that is used for pairwise comparison of subgroups, the significant difference appears between cases with rupture and thick Vernier caliper and between cases with thick and thin Vernier caliper as shown in Table 4 and Figure 3.

There is statistically positive significant correlation between CK MB and CK in CS group $(p .<0.05)$ (Figure 4). At a cut off level of $\mathrm{CK} \mathrm{MB}>24.3 \mathrm{U} / \mathrm{L}$, there is statistically significant predictive value for prediction of thin scar $(p<0.05)$ with sensitivity $100 \%$ and specificity $84.09 \%$. At a cut off level of CK MB $>27.3 \mathrm{U} / \mathrm{L}$, there is statistically significant predictive value for prediction of rupture uterus $(p .<0.05)$ with sensitivity $100 \%$ and specificity $78 \%$ (Figures 5 and 6).

Table 1: Gravidity distribution between both groups

\begin{tabular}{ccccc}
\hline & \multicolumn{2}{c}{ CS group } & \multicolumn{2}{c}{ NVD group } \\
\cline { 2 - 5 } Gravidity & $\mathrm{N}$ & $\%$ & $\mathrm{~N}$ & $\%$ \\
\hline G0 & 0 & 0 & 3 & 10 \\
G2 & 21 & & & \\
G3 & 22 & 35 & 10 & 33.3 \\
G4 & 10 & 36.7 & 6 & 20 \\
G5 & 3 & 16.7 & 5 & 16.7 \\
G6 & 3 & 5 & 4 & 13.3 \\
G7 & 1 & 5 & 1 & 3.3 \\
& & & & \\
& & & & \\
\end{tabular}

Table 2: Scar integrity in CS group

\begin{tabular}{lcc}
\hline \multirow{2}{*}{ Scar integrity } & \multicolumn{2}{c}{ CS group } \\
\cline { 2 - 3 } & $\mathrm{N}$ & $\%$ \\
\hline Defect & 4 & 6.7 \\
Intact & 39 & 65 \\
Thick & 1 & 1.7 \\
Thin & 16 & 26.7 \\
\hline
\end{tabular}

Fig. 1: Level of CKMB in vaginal delivery and cesarean delivery

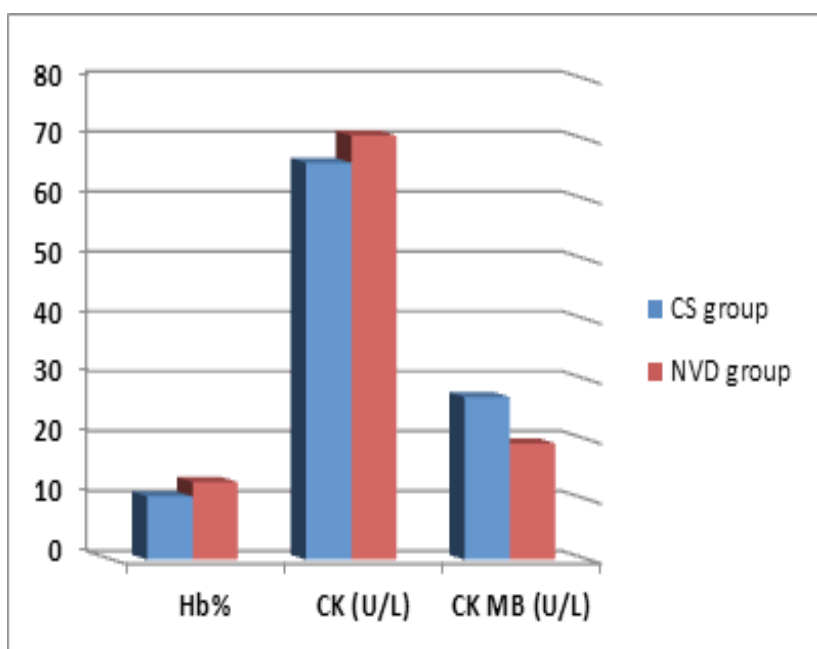

Table 3: Relation between CKMB and scar integrity

\begin{tabular}{llcl}
\hline \multirow{2}{*}{$\begin{array}{l}\text { Scar integrity } \\
\text { (CS group) }\end{array}$} & \multicolumn{2}{c}{ CK MB } & P.value \\
\cline { 2 - 3 } (1) Defect & 39.60 & 9.02 & \\
(2) Intact & 19.04 & 13.19 & $\begin{array}{c}<0.001 \\
(1) \text { and (2) }\end{array}$ \\
(3) Thick & 18.70 & & $(2)$ and (4) \\
(4) Thin & 44.39 & 13.82 & \\
\hline
\end{tabular}

Fig.2: Relation between CKMB and scar integrity in CS group by ANOVA test

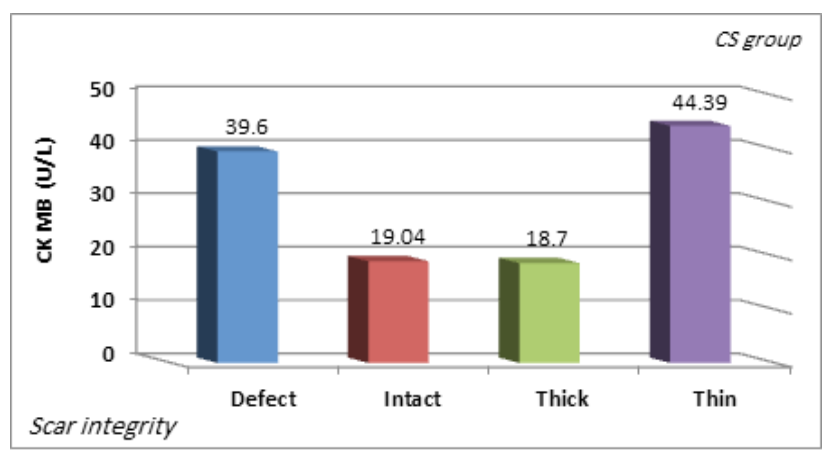

Table 4: Relation between CKMB and scar integrity in CS group by ANOVA test

\begin{tabular}{lccc}
\hline $\begin{array}{c}\text { Vernier caliper } \\
\text { (CS group) }\end{array}$ & Mean & SD & P.value \\
\hline (1) Rupture & 41.13 & 8.27 & $<0.001$ \\
(2) Thick & 19.96 & 14.18 & (1) and (2) \\
(3) Thin & 43.63 & 15.21 & (2) and (3) \\
\hline
\end{tabular}


Fig. 3: Relation between $\mathrm{CKMB}$ and scar integrity in CS group by ANOVA test

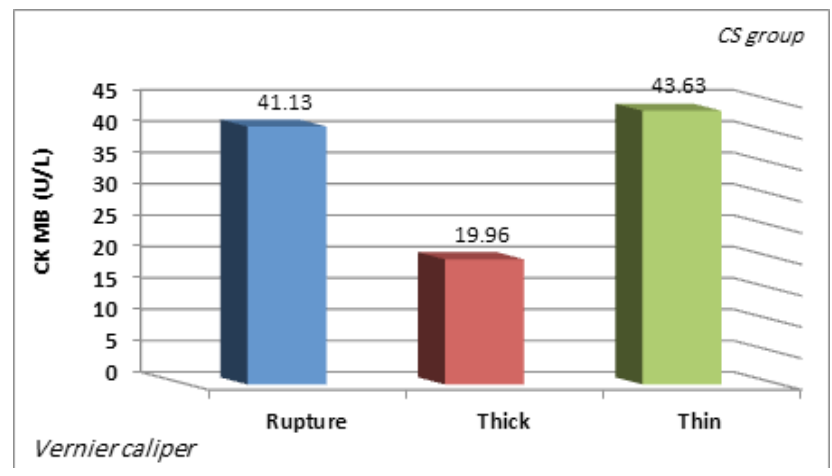

Fig. 4: Relation between CK and and CKMB in CS group

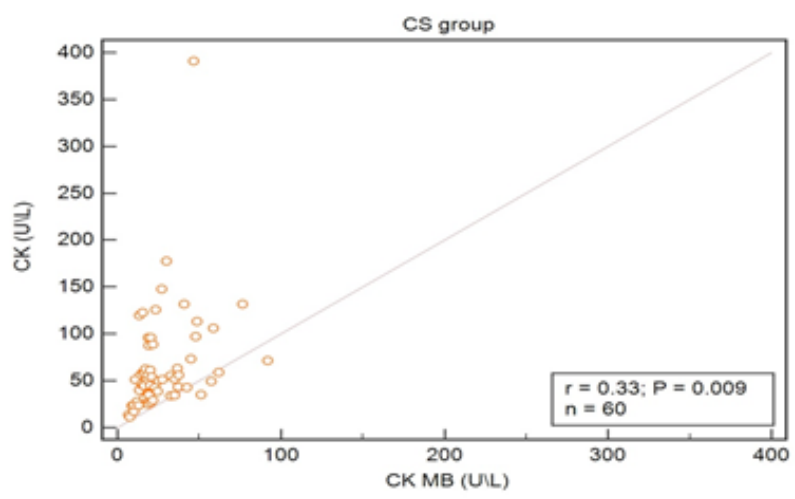

Fig. 5: CKMB as a marker of thin scar

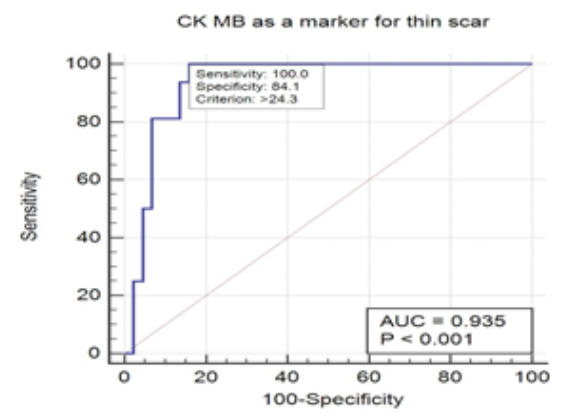

Fig. 6: $C K M B$ as a marker of rapture uterus

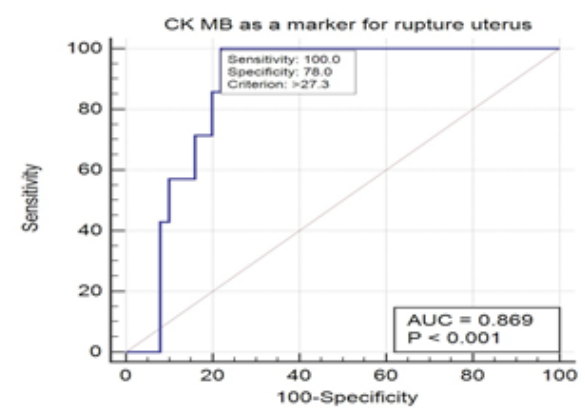

\section{DISCUSSION}

The rapid increase in caesarean birth rates from 1996 to 2011 without clear evidence of concomitant decreases in maternal or neonatal morbidity or mortality raises significant concern that caesarean delivery is overused $^{[7]}$. With this significant increase in the number of cesarean deliveries there were increases of the number of complications, one of these complications cesarean scar defect (CSD) is the most common but also the most neglected. In pregnant patients with a history of prior cesarean delivery, a severe CSD is a risk factor for both early (i.e., uterine rupture) and for late complications (i.e., ectopic pregnancy at the scar level and other scar-related abnormalities), also uterine dehiscence or rupture in future pregnancy ${ }^{[8]}$.

Diagnosis of complete uterine rupture was underreported in the electronic patient record system (EPRS) by 35\% and diagnosis of uterine dehiscence was missing in $100 \%$ of cases $^{[9]}$, Frequency of lower uterine segment (LUS) scar dehiscence is reported to be similar to the uterine rupture during labor in women with unscarred uterus. Unsecure prediction of the integrity of the scarred LUS during labor appears to be one of the reasons for high repeat cesarean rates, But there are variation between two observations by sonographic measurement of LUS is believed to be due to contractile state of the uterus, displacement of amniotic fluid, fetal movement, compression of LUS by fetus, and degree of bladder filling.Inter-operator variability is taken care of by only one sonologist performing the measurements. The operator pressure through transducer can add to some difference in the measurements ${ }^{[10]}$.

The creatine kinase is considered as to be as key enzymes of muscular metabolism. Accordingly, most of its activity is concentrated within muscular organs. Indeed the enzyme can constitute up to $20 \%$ of the soluble sarcoplasmic protein in some types of muscle. In view of these facts we can use it as laboratory parameters for muscular damage. Interest has centered on the CKMB hybrid of myocardial origin ${ }^{[11,12]}$. The uterus and placenta, two organs which were reported to embody substantial amounts of these enzymes, and which participate actively in the process of labor, are thought to release these enzymes to the circulation during labor ${ }^{[13]}$, Therefore, prediction of uterine dehiscence prior to onset of labour by using CKMB as a soft marker may have clinical significance; A prospective study performed at Al-Zahraa University Hospital where ninety women were included in our study, sixty women with previous cesarean section with forty two with elective CS and eighteen with emergency CS, thirty with NVD in active labour ranged from 18-40 years, the gravidity ranged from $0-7$. Estimated gestational age ranged from 36-40 wks, The interdelivery interval ranged 
from 12-48 months, The whole LUS thickness by 2D TAS ranged from $(2.5-5.7 \mathrm{~mm})$.

In our study, we found that there is significant increase of mean CKMB in CS group with thin and raptured scar in comparisons with NVD group in active labour which was normal $(p<0.05)$. Soundravally et al, reported that the elevated CK-MB during pregnancy should not be due myocardial infarction for CK-MB is found not only in myocardium but also in uterus and placenta ${ }^{[14]}$, Our theory that CKMB may release from scarred uterus before delivery or from myometrial tissue or from area of dehiscence as this area subjected to ischemia .even though none of our patient had cardiac symptoms or complications and this may give idea about scar dehiscence or rapture. As CKMB mostly of muscle origin and reflecting labour stress or injury ${ }^{[14]}$.

This may support our theory as some research shows that rising level of CK occurs as a result of muscle tissue damage. Additionally, cesarean section known to increase CK levels. Also the destruction of the myometrial tissue causes serum CK increase. Turan and his coworkers measured CK levels to investigate if the locked method gave injury to the uterine muscle. The increase in the levels of $\mathrm{CK}$ in locked method was remarkable ${ }^{[15]}$, Researchers believe that serum $\mathrm{CK}$ can be a diagnostic marker of tubal ectopic pregnancy (tEP), as the theory on which it is claimed that $\mathrm{CK}$ can be marker of tEP is that the tubal musculature lacks a submucosal layer and hence the penetrating trophoblastic tissue is expected to injure the tubal smooth muscle and release a sufficient amount of this enzyme into circulation ${ }^{[13]}$, Also serum CPK activity were reported significantly higher in women with tubal pregnancy that may or may not have ruptured and are reliable in the diagnosis of a tubal pregnancy. They observed significant elevation in CPK and CKMB serum activity in ectopic pregnancy ${ }^{[13]}$, Shao and Yang used creatine kinase (CK) in the diagnosis of scar uterus and placenta previa implantation, CK expression in the implantation group were,significantly higher than non-implantation group ${ }^{[16]}$, This is may be explained by Banu et al, who suggested that an increasing amount of serum creatine kinase (CK) isoenzymes would give an idea about the destruction of the myometrial tissue $\mathrm{e}^{[17]}$

Our results did not matche McNeely and his coworkers who measured serum CK activity of 28 women with normal pregnancy at six time interval; during the third trimester, between 30 and 36 weeks gestation, on admission to hospital in labor, within $30 \mathrm{~mm}$ after delivery, between 7 and $9 \mathrm{~h}$ after delivery, on the fifth day postpartum and at the time of the routine six-week check-up. Mean CK activity were $29,45,109,132,49$ and $35 \mathrm{U} / \mathrm{L}$ at 1,2,3,4,5 and 6 time interval. Thus, showing that there is transit rise in CK activity as soon as time of delivery approaches and peak activity are obtained between 7 to $9 \mathrm{hrs}$ after the delivery. The $\mathrm{CK}$ activity tends to reach at normal levels within six-weeks after delivery. Similar trends were observed for the CK isoenzymes CKMB activity, there is significant association between serum CKMB activity and route of delivery ${ }^{[12,5]}$. Also, did not matches Leiserowitz and his coworkers who conducted his study on forty-nine normal pregnant women recruited late in the third trimester for serial determinations of creatine kinase (CK) and its $\mathrm{MB}$ isoenzyme fraction (CK-MB) at four different times; on recruitment between 36 and 40 weeks gestation, on admission in active labor, immediately after delivery and on the first postpartum day. In the patients with vaginal delivery total $\mathrm{CK}$ was significantly elevated at time 4 compared with times 1, 2 and 3 (Pvalue $<0.0001$ ). CK-MB fraction was also significantly elevated at time 4 compared with times 1, 2 and 3 ( $P$ value $<0.0001)$. In $35.7 \%$ of the patients at time $4, \mathrm{CK}-\mathrm{MB}$ was sufficiently elevated to give the laboratory interpretation of "borderline" or "consistent with a myocardial infarction," even though none of the patients had cardiac symptoms or complications ${ }^{[5]}$, But this not matches Torabi and coworkers: who found lower CK$\mathrm{MB}$ in cesarean section than NVD.CK-MB isoenzymes levels in newborns delivered vaginally were higher than elective cesarean section. This may be CKMB were taken from neonates serum not from the mothers so it did not match our theory ${ }^{[18]}$.

Also, this may support our theory that area of dehiscence undergo ischemia that release markers of ischemia $\left(\mathrm{CPK}, \mathrm{K}^{+}, \mathrm{Ca}^{2+}\right)$ this may explained by $\mathrm{I} / \mathrm{R}$ (ischemia reperfusion theory while putting tourniquet at time of removal of myoma. These data assume that surgeries requiring any temporary arrest of the arterial circulatory system, such as tourniquet use, lead to ischemia reperfusion and apoptosis. This IR would itself be responsible for an increase marker of ischemia $\left(\mathrm{CPK}, \mathrm{K}^{+}\right.$, and $\left.\mathrm{Ca}^{+}\right)$. Oksuzoglu et al, comparing plasma levels of CPK and interleukin-6 (IL6) in different types of uterine surgery, found higher levels of these markers during uterine abdominal versus laparoscopic surger ${ }^{[19]}$. Khan and Rathore (2017) also concluded that the rise of total CK and $\mathrm{CK}-\mathrm{MB}$ in maternal serum was directly correlated with the type of delivery also lower segment cesarean section LSCS mode of delivery is associated with increased CKMB $\operatorname{activity}^{[5]}$.

In our study, we found there was relation between CKMB and intraoperative scar grading was determined. And showed a significant relation of mean CK MB in CS group according to scar integrity distribution by ANOVA test $(p<0.05)$. By doing Student-Newman-Keuls test; Posthoc test that is used for pairwise comparison of subgroups, the significant difference appears between cases with defect and intact scar and between cases with intact and thin scar (i.e. the patients with thin and defect scar have elevated levels of CKMB, while patients with intact scar have normal level of CKMB). Also this confirmed 
by using Vernier caliper intraoperative which showed significant relation of mean CK MB in CS group according to Vernier caliper distribution by ANOVA test $(p<0.05)$, the significant difference appears between cases with rupture and thick Vernier caliper and between cases with thick and thin Vernier caliper (i.e. the patients which proved to be thin and defect scar by Vernier caliper have elevated level of CKMB and patients which were thick or intact scar have normal levels of CKMB).

And most of the significance of CKMB and prediction of scar integrity in cases with G3 and G2. There is statistically significant difference of mean CK MB in CS group according to gravidity distribution by ANOVA test $(p<0.05)$. By doing Student-Newman-Keuls test; Post-hoc test that is used for pairwise comparison of subgroups, the significant difference appears between cases with G2 and cases with G3 (i.e. most patients with G2 (P1CS), G3 (P2CS) were with elevated level of CKMB in comparisons with other gravidity) this may be attributed to most cases were G2 (P1CS 23 cases), G3 (P2CS 27 cases) and this may be related more to scar dehiscence itself not gravidity or parity. Also we did not find any significance between interdelivery interval and scar dehiscence as CKMB not affected if interdelivery interval was less or more than 24 months as it was affected by dehiscence this may be related to our small sample size. As Bujold et al. reported that $10.5 \%$ of dehiscence occurred in patients with interdelivery interval of less than 24 months compared to $3 \%$ after 24 months. This finding may be related to the time needed for proper scar healing. Using magnetic resonance imaging, Dicle et al. showed that uterine scars needed at least 6 months to reach a normal appearance ${ }^{[20]}$.

There is statistically no significant difference of mean CK MB in CS group according to pulse or scar tenderness distribution by ANOVA test ( $p$.> 0.05) (i.e. pulse or scar tenderness not indicative of scar dehiscence and CKMB was normal in these cases).This in agreement with Ramadan et al., who could not find an association between having pain at the cesarean scar and the risk for developing dehiscence. Similar observations were reported by Suzuki et al., who concluded that there was no increased rate of dehiscence in patients who felt pain and tenderness in the lower uterine segment ${ }^{[20]}$. We did not find any correlation between $\mathrm{CKMB}$ and uterine contraction as CKMB not elevated with the patients have uterine contraction or in labour and these patients during $\mathrm{CD}$ had not dehiscence in their cesarean scar (no significant difference of mean $\mathrm{CK} \mathrm{MB}$ in $\mathrm{CS}$ group according to presence of contraction by ANOVA test $(p .>0.05)$. This in agreement with Ramadan et al, who found the presence of labor at the time of CD was noted to have no significant effect on the rate of dehiscence ${ }^{[20]}$. May be there association between myocardium and myometrium isoenzymes as in non-controlled preliminary rabbit experiments indicate that patches of uterine myometrium reinforced by greater omentum can be used as autologous transplant therapy for infracted myocardium ${ }^{[21]}$.

Therefore. we can use CKMB as a soft marker beside other methods as LUS thickness by U/S to predict dehiscence scar before CS also before taking decision of TOLAC. At a cut off level of CK MB $>24.3 \mathrm{U} / \mathrm{L}$, there is statistically significant predictive value for prediction of thin scar $(p<0.05)$ with sensitivity $100 \%$ and specificity $84.09 \%$. And at a cut off level of $\mathrm{CK} \mathrm{MB}>27.3 \mathrm{U} / \mathrm{L}$, there is statistically significant predictive value for prediction of rupture uterus $(p<0.05)$ with sensitivity $100 \%$ and specificity $78 \%$.

Our limitation small sample size and most of our sample size were G2, G3, but this the first paper talking about CKMB and its relation to CS scar complication prior to delivery.

\section{CONCLUSION}

We can use CKMB as a soft marker beside other method for detection of cesarean section complication before delivery as it is cheap, available with high sensitivity and specificity.

\section{CONFLICT OF INTERESTS}

There are no conflicts of interests.

\section{REFERENCE}

1. Molina G, WeiserTG, Lipsitz SR, Esquivel MM, UribeLeitz T, Azad T, et al.(2015): Relationship Between Cesarean Delivery Rate and Maternal and Neonatal Mortality. JAMA; 314(21):2263-70.10.1016/S08954356(96)00236-3.m.,

2. Jha, N. N. S., Maheshwari, S., \& Barala, S. (2018) Ultrasonographic assessment of strength of previous cesarean scar during pregnancy. International Journal of Reproduction, Contraception, Obstetrics and Gynecology, 7(4), 1459.

3. Al-Zirqi, I., Stray-Pedersen, B., Forsén, L., Daltveit, A. K., \& Vangen, S. (2016). Uterine rupture: trends over 40 years. BJOG: An International Journal of Obstetrics \& Gynaecology, 123(5), 780-787.

4. Asakura H, Nakai A, Ishikawa G, Suzuki S and Araki T (2000): Prediction of uterine dehiscence by measuring lower uterine segment thickness prior to the onset of labor: evaluation by transvaginal ultrasonography. J Nippon Med Sch; 67(5):352-6. 
5. Khan A, Rathore B (2017): TOTAL CREATINE KINASE AND ITS ISOENZYMES IN CORD BLOOD OF FULL TERM NEWBORNS. Era's Journal of Medical Research, 4(1), 27-38.

6. Khan A, Rathore B, Singh K, Singh M, Misra (2017): Association of creatine kinase and its isoenzymes (CK$\mathrm{MB}, \mathrm{CK}-\mathrm{BB})$ activity with high risk pregnancy. Int $\mathrm{J}$ Res Med Sci, 5(4), 1464-1470.

7. Caughey, A. B., Cahill, A. G., Guise, J. M., Rouse, D. J., \& American College of Obstetricians and Gynecologists. (2014). Safe prevention of the primary cesarean delivery. American journal of obstetrics and gynecology, 210(3), 179-193.

8. Rosa, F., Perugin, G., Schettini, D., Romano, N., Romeo, S., Podestà, R., ... \& Gandolfo, N. (2019). Imaging findings of cesarean delivery complications: cesarean scar disease and much more. Insights into imaging, 10(1), 98 .

9. Fogelberg, M., Baranov, A., Herbst, A., \& Vikhareva, O. (2017). Underreporting of complete uterine rupture and uterine dehiscence in women with previous cesarean section. The Journal of Maternal-Fetal \& Neonatal Medicine, 30(17), 2058-2061.

10. Kushtagi, P., \& Garepalli, S. (2011). Sonographic assessment of lower uterine segment at term in women with previous cesarean delivery. Archives of gynecology and obstetrics, 283(3), 455-459.

11. Lang, H. (Ed.). (2012). Creatine kinase isoenzymes: pathophysiology and clinical application. Springer Science \& Business Media.

12. McNeely, M. D., Baerris, B., Papsin, F. R., Lyons, E., \& Schipper, H. (1977). Creatine kinase and its isoenzymes in the serum of women during pregnancy and the peripartum period.Clinical chemistry, 23(10), $1878-1880$.

13. Soundravally, R., Krishna Latha, T., Soundara Raghavan, S., Ananthanarayanan, P. H., \& Srilatha, K. (2013). Diagnostic significance of total creatine kinase and its isoform in tubal ectopic pregnancy. Journal of Obstetrics and Gynaecology Research, $39(12), 1587-1591$
14. Almeida, C. M., Carrapato, M. R., Pinto, F., Pinto, M., Ferreira, S., Schmitt, D., \& Marinho, L. (2011). Biochemical markers of neonatal myocardial dysfunction. The Journal of Maternal-Fetal \& Neonatal Medicine, 24(4), 568-573.

15. Turan, G. A., Gur, E. B., Tatar, S., Gokduman, A., \& Guclu, S. (2014). Uterine closure with unlocked suture in cesarean section: Safety and Quality. Pakistan journal of medical sciences, 30(3), 530 .

16. Shao, Y., \& Yang, S. (2018). The diagnostic value of two-dimensional ultrasound and three-dimensional color Doppler ultrasonography combined with AFP and CK in the diagnosis of scar uterus and placenta previa implantation. Journal of Chinese Physician, 20(5), 676-679.

17. Banu, N.S., Gaze, D.C, Bruce, H., Collinson, P.O., Belli, A.M., \& Manyonda, I.T. (2007): Markers of muscle ischemia, necrosis, and inflammation following uterine artery embolization in the treatment of symptomatic uterine fibroids. American journal of Obstetrics and gynecology, 196(3), 213-e1.

18. Torabi, Z., Salmani, R., Fayaz, A., \& Ahmadiafshar, A. (2014). Comparative levels of total creatine kinase and MB-isoenzyme in cord blood between vaginal delivery and cesarean section. International Journal of Biochemistry Research \& Review, 4(4), 306-311.

19. Essone, J. N., Abessolo, F. O., Tsagoulela, A., Nkhilli, G. E., Assoumou, P., Ntamack, J. B., ... \& Milama, E. N. (2019). Uterine Reperfusion Ischemia and Use of Elastic Tourniquet.Journal of Biosciences and Medicines, 7(2), 98-119.

20. Ramadan, M. K., Kassem, S., Itani, S., Sinno, L., Hussein, S., Chahin, R., \& Badr, D. A. (2018). Incidence and Risk Factors of Uterine Scar Dehiscence Identified at Elective Repeat Cesarean Delivery: A Case-Control Study. Journal of Clinical Gynecology and Obstetrics, 7(2), 37-42.

21. Taheri, S. A., Yeh, J., Batt, R. E., Fang, Y., Ashraf, H., Heffner, R., ... \& Naughton, J. (2008). Uterine myometrium as a cell patch as an alternative graft for transplantation to infarcted cardiac myocardium: a preliminary study. The International journal of artificial organs, 31(1), 62-67. 